\title{
Exkurs: Intensivtherapie des pädiatrischen Organspenders
}

\author{
Klaus Michael Lücking, Kathrin Seidemann und Florian Hoffmann
}

Die Neuausrichtung des übergeordneten Therapieziels (Janssens et al. 2013) bei Patienten mit deletärer Hirnschädigung - von einem primär kurativen Therapieziel hin zu einem neuen Therapieziel „Organspende“ - findet ihren Niederschlag auch in der konkreten hands-on-Intensivtherapie: Die bisherige und mit kurativem Therapieziel initiierte Intensivtherapie soll weiterhin organprotektiv und konsequent leitliniengerecht fortgeführt werden - mit Ausnahme der nun nicht mehr notwendigen Cerebroprotektion, nachdem die Hirnschädigung als prognostisch infaust eingeschätzt worden ist. Der intensivmedizinische Fokus liegt nun auf dem Erhalt der metabolischen Homöostase und dem Funktionserhalt, ggf. sogar der Funktionsoptimierung potentiell transplantabler Organe. Die Leitlinien verschiedener pädiatrischer Fachgesellschaften und nationaler Organspende-Organisationen geben (ähnlich wie in der Diagnostik des irreversiblen Hirnfunktionsausfalles, IHA) einen nationalen Expertenconsensus wieder; Empfehlungen werden häufig als „Good clinical practise“ aus Erwachsenenstudien (mit ggf. höherem Evidenzgrad) abgeleitet (Kotloff et al. 2015, Nakagawa et al. 2018, NHS Blood and Transplant 2018, Riedel u. Rimensberger 2020, Vyas u. Nakagawa 2020).

Die cerebrale Einklemmung mit konsekutiv globalem Hirnfunktionsausfall führt zu (in der Regel passageren) Funktionseinschränkungen extra-cerebraler Organe. Die Kenntnis und Antizipation der häufigen pathophysiologischen Veränderungen und deren Auswirkungen auf die verschiedenen Organsysteme ist die Voraussetzung für eine zeitnahe und zielgerichtete 
Korrektur. Sie trägt damit entscheidend zum Erfolg der Organspende mit schlussendlich guter Transplantatfunktion im Empfänger bei. Die Behandlung des potentiellen Organspenders folgt deshalb etablierten pädiatrischintensivmedizinischen Therapieprinzipien. Hierbei sind neben der Berücksichtigung altersabhängiger Normwerte und gewichtsabhängiger Dosierungsvorgaben auch konzeptionelle Besonderheiten in der pädiatrisch-neonatologischen Intensivmedizin $\mathrm{zu}$ beachten, die im Folgenden für die jeweiligen Organsysteme skizziert werden.

\section{Kardiovaskuläres System}

Einer passageren arteriellen Hypertension (Cushing-Trias, „Katecholaminsturm“ oder „autonomic storm“) im Rahmen der finalen cerebralen Einklemmung folgt häufig ein ausgeprägter Tonusverlust der systemarteriellen Widerstandsgefäße mit konsekutivem Blutdruckabfall. Angesichts der Paralyse der autonomen Regulation sistiert auch die reflektorische Tachykardie zur Steigerung des Herz-Zeit-Volumens (HZV). Ein (häufig passageres) myokardiales Pumpversagen (Tako-tsubo-like-Kardiomyopathie) trägt ggf. zur Kreislaufinsuffizienz bei, ebenso ein Volumenverlust im Rahmen eines nicht diagnostizierten oder unzureichend behandelten Diabetes insipidus centralis. Essentiell für eine differenzierte Kreislauftherapie sind - neben dem Basismonitoring bestehend aus EKG, transkutaner Sauerstoffsättigungsmessung, kontinuierlicher Messung der Körperkerntemperatur sowie kontinuierlicher Ein- und Ausfuhrkontrolle - ein engmaschiges hämodynamisches Monitoring mit kontinuierlicher invasiver arterieller und zentralvenöser Drucküberwachung, arteriellen und gemischtvenösen Blutgasanalysen (Beurteilung von Lactat und arteriovenöser Sättigungsdifferenz $\mathrm{avDO}_{2}$ als indirekte Parameter für Sauerstoffausschöpfung im Gewebe und metabolische Homöostase) sowie seriellen echokardiographischen Untersuchungen zur Beurteilung der myokardialen Pumpfunktion. Ein erweitertes invasives hämodynamisches Monitoring (z.B. HZV-Messung mittels PICCO, Pulmonaliskatheter u.a.) kann erwogen werden, zeigt allerdings zumindest unter Studienbedingungen keinen zusätzlichen Vorteil für die Organspende oder die Transplantatfunktion (Al-Khafaji et al. 2015)

Die Volumentherapie mit balancierten Vollelektrolyt-Lösungen (Blut- und Plasmabestandteile nur nach strenger Indikation) zur Stabilisierung des Blutdrucks sollte eher restriktiv gestaltet werden, insbesondere bei möglicher Lungenspende, aber auch zur Vermeidung einer progredienten Schwellung abdomineller Organe mit dem Risiko der Entwicklung eines abdominellen Kompartmentsyndroms. Als Vasopressoren werden (bei angemessener kardialer Pumpfunktion) in Europa initial häufig Norepinephrin und 
bei therapierefraktärer Vasoplegie auch Vasopressin eingesetzt; letzteres insbesondere dann, wenn neben dem Gefäßwiderstandsverlust auch ein behandlungsbedürftiger Diabetes insipidus centralis vorliegt. Ist die myokardiale Pumpfunktion kompromittiert, so ist eine positiv inotrope Therapie mit Dobutamin oder Epinephrin oder Milrinon indiziert, bei letzterem kann eine Kombination mit niedrig dosiertem Norepinephrin sinnvoll sein. Auch der Einsatz von Levosimendan kann erwogen werden, allerdings fehlt hierfür bisher der evidenzbasierte Nachweis eines positiven Effekts auf die Transplantatfunktion. Bleibt die echokardiographisch ermittelte Ejektionsfraktion $(\mathrm{EF})$ trotz eines angemessenen Flüssigkeitsregimes und positiver Inotropie bei $<45 \%$, so wird die Substitution von aktivem Schilddrüsenhormon (Liothyronin) empfohlen. Die Empfehlungen zu einer frühzeitigen Hydrocortisonsubstitution (Dosierung $100 \mathrm{mg} / \mathrm{m}^{2} \mathrm{KOF} / \mathrm{d}$ ) sind zwar widersprüchlich, angesichts der unterstellten Unterbrechung der hypothalamisch-hypophysären Achse mit konsekutiver Nebenniereninsuffizienz erscheint diese bei katecholaminpflichtigem Kreislaufversagen jedoch gerechtfertigt und hat sich allgemein etabliert.

Steht die Zytokin- und Katecholaminsturm-assoziierte Tachykardie im Vordergrund (bei noch weitgehend unbeeinträchtigter systolischer Pumpfunktion), so kann zur Senkung der Herzfrequenz und damit Stabilisierung der koronaren Perfusion eine $\beta$-Blockertherapie erwogen werden. Esmolol (Dosierung $2 \times 1 \mu \mathrm{g} / \mathrm{kg} / \mathrm{min}$ ) hat sich wegen seiner guten Steuerbarkeit und seines relativ geringen Einflusses auf die diastolischen Blutdrücke bewährt. Prädisponierende Faktoren für kardiale Arrhythmien, wie myokardiale Ischämie, Imbalancen im Elektrolyt- und Säure-Basen-Haushalt, Hypothermie sowie Wahl und Dosierung der Katecholamintherapie prädisponieren für kardiale Arrhythmien. Eine neu auftretende Bradykardie ist in der Regel Atropin-resistent (der inhibitorische vagale Einfluss ist erloschen!), sodass hier Dobutamin, bei stabilem Kreislauf auch Isoproterol, zur Steigerung der Herzfrequenz empfohlen werden können.

\section{Respiratorisches System}

Eine Lungen-protektive Beatmung ist eine entscheidende Säule der Intensivtherapie des kindlichen Organspenders und hat nicht nur Auswirkungen auf Qualität und Zahl potentieller Lungenspenden, sondern auch auf Hämodynamik und Homöostase anderer potentiell transplantabler Organe: Nach Rekrutierungsmanöver und PEEP-Optimierung sollte eine high-PEEP/lowtidal-volume-Beatmung mit Tidalvolumina von $5-6 \mathrm{ml} / \mathrm{kg} \mathrm{KG}$ angestrebt werden. Die Spitzen- bzw. Plateaudrücke sollten dabei < 28-30 $\mathrm{cm} \mathrm{H}_{2} \mathrm{O}$ liegen bei einem delta-p von maximal $15 \mathrm{~cm} \mathrm{H}_{2} \mathrm{O}$ und einem $\mathrm{FiO}_{2}$ von $<0,5$ zur 
Reduktion der Sauerstofftoxizität durch intrapulmonale Radikalbildung. Eine permissive Hyperkapnie ist nun (nach Beendigung einer den gesteigerten Hirndruck adressierenden Beatmungsstrategie) wieder möglich, sofern die Homöostase des Patienten (Ziel-pH > 7,25) und der pulmonal-arterielle Druck dies erlauben. Tägliche Rekrutierungsmanöver sind fester Bestandteil der Lungenprotektion des erwachsenen Organspenders und können auch bei pädiatrischen Patienten durchgeführt werden; Lagerungstherapie zur Vermeidung von Atelektasen, engmaschige Physiotherapie und ggf. repetitive Bronchoskopien (letztere auch zur Erregersuche und gezielter antibiotischer Therapie) sind weitere Bausteine einer konsequent durchgeführten lungenprotektiven Respirator-Therapie.

\section{Endokrines und metabolisches System}

Störungen der hypothalamisch-hypophysären Achse zeigen sich häufig (in ca. 40\% der pädiatrischen Patienten) als Diabetes insipidus centralis, klinisch charakterisiert durch eine erheblich gesteigerte Diurese von wasserklarem Urin mit deutlich reduziertem Urin-Natrium und Urin-Osmolarität (spezifisches Gewicht $<1.005 \mathrm{mg} / \mathrm{ml}$ ) sowie konsekutivem Anstieg des Serum-Natriums sowie der Serumosmolarität. Neben dem Volumenersatz mit ggf. halb-isotonen Lösungen ist intravenöses Desmopressin unverzichtbar (die übliche intranasale Applikation wird bei unsicherer hypophysärer Perfusion nicht empfohlen). Angloamerikanische Leitlinien empfehlen den frühzeitigen Einsatz von Vasopressin bei Vasopressorenbedarf und manifestem Diabetes insipidus centralis. Eine Überdosierung oder ein SIADH erfordern eine strikte Flüssigkeitsbilanz.

Der durch die cerebrale Einklemmung provozierte Zytokin-Sturm kann neben einer generalisierten Endothelschädigung und nachfolgendem SIRS zu einer sekundären Insulinresistenz und ausgeprägten Glucoseverwertungsstörung führen. Dennoch sollte eine altersentsprechende Clucosezufuhr (3-8 g/kg/d) angestrebt bzw. eine basale Glucosezufuhr von 2-3 g/kg/d nicht unterschritten werden. Bei anhaltender Hyperglykämie (BZ > $10 \mathrm{mmol} / \mathrm{l})$ ist eine kontinuierliche Insulintherapie indiziert. Eine enterale Ernährung sollte nicht vernachlässigt werden. Angesichts ihrer Relevanz für die Sicherung der Homöostase, aber auch die Barriere- sowie die immunmodulatorische Funktion des Darmes sollten deshalb mindestens ,trophic enteral feeds“ zur Aufrechterhaltung der Enterozytenfunktion verabreicht werden. Ist dies gastral aufgrund einer ausgeprägten Magenatonie nicht möglich, sollte die Anlage einer Duodenalsonde erfolgen. Zur Vermeidung von Dumping und starken Schwankungen der Blutzuckerspiegel sollte die Nahrungszufuhr dann kontinuierlich über eine Pumpe erfolgen. 
Die häufig dysregulierte diencephale Steuerung der Körperkerntemperatur bedarf einer konsequenten Temperaturkontrolle durch externe physikalische Maßnahmen, z.B. durch Einsatz von Kühlmatten mit automatischer Temperatursteuerung. Ziel ist derzeit strikte Normothermie $\left(36-37,5^{\circ} \mathrm{C}\right)$. Für die Diagnostik des irreversiblen Hirnfunktionsausfalles ist eine Körperkerntemperatur von $\geq 35^{\circ} \mathrm{C}$ vorgesehen. Für eine milde systemische Hypothermie nach IHA-Feststellung zur Verbesserung des Transplantations-Outcomes gibt es Evidenz, bisher aber keinen internationalen Konsens (Malinoski et al. 2019).

\section{Erweiterter intensivmedizinischer Behandlungsbedarf}

Der Einsatz extracorporaler Organersatzverfahren ist grundsätzlich keine Kontraindikation für eine Organspende. Auch nach protrahierter Reanimation und ggf. Implantation einer ECLS ist trotz initialer schwerster Hypoxämie eine erfolgreiche Organprotektion mit folgender Organspende möglich. Gerade hier kann die konsequente Fortführung einer organ-protektiven Intensivtherapie zu einer vollständigen Erholung der Organfunktion innerhalb weniger Tage mit anschließend guten Transplantationsergebnissen führen. Auch die Diagnose des IHA ist selbst unter laufender va-ECMO/ECLS möglich, wenngleich nicht trivial. Die Reanimation des kindlichen Organspenders bedarf ebenso wie eine Eskalation der Intensivtherapie auf dem Weg zur Organspende (bis hin zur sekundären Anlage extracorporaler Verfahren oder operativer Eingriffe) einer sorgfältigen und vorausschauenden Abwägung - nicht nur im Team, sondern auch mit den Eltern und Sorgeberechtigten des Patienten. Ein aktuelles Positionspapier der DIVI-Sektionen Ethik und Organspende und -transplantation gibt Hilfestellungen in diesen komplexen Entscheidungsprozessen (Neitzke et al. 2019).

Fazit

- Die Intensivtherapie des kindlichen Organspenders orientiert sich an bekannten pädiatrisch-intensivmedizinischen Konzepten; sie sollte in jedem Fall konsequent und leitliniengerecht umgesetzt werden.

- Passager geschädigte, aber prinzipiell transplantable Organe können sich unter einer konsequenten Organprotektion innerhalb weniger Tage funktionell erholen.

- Der Einsatz extracorporaler Organersatzverfahren, aber auch anderer invasiver Eingriffe, bedarf - wie auch eine Entscheidung zur kardiopulmonalen Reanimation - der vorausschauenden Abwägung und Konsentierung im interprofessionellen Team wie mit den Sorgeberechtigten. 


\section{Literatur}

Al-Khafaji A, Elder M, Lebovitz DJ et al. (2015) Protocolized fluid therapy in brain-dead donors: the multicenter randomized MOnIToR trial. Intensive Care Med 41(3):418-26

Janssens U, Burchardi H, Duttge $G$ et al. (2013) Therapiezieländerung und Therapiebegrenzung in der Intensivmedizin.Positionspapier der Sektion Ethik der DIVI. Anaesthesist 62(1):47-52

Kotloff RM, Blosser S, Fulda G/ et al. (2015) Management of the Potential Organ Donor in the ICU: Society of Critical Care Medicine/American College of Chest Physicians/Association of Organ Procurement Organizations Consensus Statement.Crit Care Med 43(6):1291-1325

Malinoski D, Patel MS, Axelrod DA et al. (2019) Therapeutic Hypothermia in Organ Donors: Follow-up and Safety Analysis. Transplantation 103 (11): e365-e368

Nakagawa TA, Shemie SD, Dryden-Palmer K et al. (2018) Organ Donation Following Neurologic and Circulatory Determination of Death. Pediatr Crit Care Med 19(8S Suppl 2):S26-S32

Neitzke G, Rogge A, Lücking KM et al. (2019) Entscheidungshilfe bei erweitertem intensivmedizinischem Behandlungsbedarf auf dem Weg zur Organspende. Positionspapier der Sektion Ethik und der Sektion Organspende und -transplantation der Deutschen Interdisziplinären Vereinigung für Intensiv- und Notfallmedizin (DIVI) unter Mitarbeit der Sektion Ethik der Deutschen Gesellschaft für Internistische Intensivmedizin und Notfallmedizin (DGIIN). Med Klin Intensivmed Notfmed 114:319-326.114

NHS Blood and Transplant (2018) Integrated Care Plan for Organ \& Tissue Donation. URL: https:// www.odt.nhs.uk/deceased-donation/best-practice-guidance/paediatric-care/ (abgerufen am 23.07.2021)

Riedel T, Rimensberger P (2020) The Swiss Donation Pathway - Modul 4: Behandlung des Pädiatrischen Spenders. URL: https://www.swisstransplant.org/de/infos-material/fuer-fachpersonen/ swiss-donation-pathway/ (abgerufen am 23.07.2021)

Vyas H, Nakagawa TA (2020) Management of the potential pediatric organ donor. URL: https://www. uptodate.com/contents/management-of-the-potential-pediatric-organ-donor (abgerufen am 23.07.2021) 